\title{
From the journals
}

\section{Resistance of $\boldsymbol{H}$ influenzae to amoxycillin}

A recent study from the UK has looked at the resistance of Haemophilus influenzae to amoxycillin in patients who had been treated in the community with the drug in the preceding 3 months for respiratory tract infections and subsequently needed hospitalisation. When data from 412 adults were analysed 73 isolates (17.7\%) were resistant to amoxycillin. This study has indentified recent hospitalisation and community prescription of amoxycillin as risk factors for amoxycillin resistance in $H$. influenzae. Journal of Antimicrobial Chemotherapy 2000; 46: 307-9. H.influenzae is a common pathogen in bronchitis, pneumonia, otitis media and meningitis. It is also a commensal in the upper respiratory passages in adults and children. Hence, acquisition of resistance to amoxycillin by this common organism could have serious implications. These findings reiterate the importance of having control measures to reduce the inappropriate use of antimicrobials in the community and in hospitals.

\section{Lipid lowering drugs for primary prevention of coronary heart disease}

Although the effectiveness of lipid lowering agents for secondary prevention in people with lipid disorders is strongly supported by clinical trials, primary prevention trials amd systematic reviews have reached mixed conclusions. An updated meta-analysis shows that use of lipid lowering drugs for primary prevention reduces the relative risk of coronary heart disease events and mortality from coronary heart disease by about $30 \%$. However, the all-cause mortality is not reduced. The failure of drug treatment to reduce all-cause mortality in primary prevention is most likely to be due to the generally low risk of mortality in the study populations. Studies of people with a high risk may reduce all-cause mortality whereas studies in subjects at low risk may not do so. Also, if low risk populations were treated for longer periods significant reductions in all-cause mortality may be achieved. Presently there is insufficient data on people at low levels of risk. Most trials have enrolled middle-aged European men. Further trials on people of non-European descent, women and the elderly, and the effect of longer treatment (5 to 10 years) have to be studied. British Medical Journal 2000; 321: 9836.

\section{Litigation in surgical practice in the UK}

All operations carry risks to the patient. Often these risks are known and they should be explained pre-operatively. Most complications ending up in litigation are preventable and arise because of failure to follow clear systems and clear procedures or due to some intrinsic fault within the procedure itself. Examples include issues of consent, retained items, surgery on the wrong side, wrong procedure, unnecessary surgery and diathermy injury. Often several factors contribute to patient harm. The Medical Defence Union of the United Kingdom has identified common and recurring themes in settlement cases. These include communication failure between professionals and between doctors and patients, inadequate clinical record keeping, poor training and inadequate continuing professional development, and system failure. British Journal of Surgery 2000; 87: 977-9. Although we do not have as much medical litigation as in the West, recognising the risks associated with surgery is essential in implementing strategies to reduce risk and improve patient safety.

\section{Risk factors for sepsis in surgical patients}

Patient related factors represent the greatest risk for development of post-operative sepsis, rather than factors associated with the surgery. Coma during 48 hours before surgery, low serum albumin level at admission, presence of two or more intrinsic comorbidities and parenteral nutrition were the main risk factors for sepsis. The variables that were related to surgery that increased risk of sepsis were emergency surgery, abdominal surgery, and the number of surgical interventions. Identifying the patients at high risk of developing post-operative sepsis, and prevention and treatment of predisposing conditions (such a coma or malnutrition) will contribute to improved patient prognosis. British Journal of Surgery 2000; 87: 1076-81. 


\title{
Identifying those at risk of post-operative pulmonary embolism after gynaecologic surgery
}

Post-operative pulmonary embolism is an important cause of morbidity and mortality. Because of its sudden and unexpected appearance the post-operative course of some patients who are otherwise recovering well is changed dramatically. If methods are available which can detect patients at risk of getting this complication such methods would be useful in patient management. A recent study from Japan where they studied women undergoing gynaecological surgery has shown that patients at risk of pulmonary embolism could be identified by ultrasound assessment of the abdominal wall fat index. They found that women with hypertension and an abdominal wall fat index value over 0.85 are at high risk of developing post-operative pulmonary embolism. Low dose heparin is indicated in such patients. International Journal of Gynaecology and Obstetrics 2000; 68: 241-7.

\section{Efficacy and safety of Norplant}

A 10-year follow up study from China about the contraceptive implant Norplant has shown it to be extremely effective and safe for long term use. This study which had 1657 women had cumulative pregnancy rates of $0.05 \%, 0.1 \%$ and $0.7 \%$ respectively at 1,3 and 5 years. The rates of menstrual disturbances peaked at 3 months (75\%) and decreased consistently to $20 \%$ at 5 years. Compared to the first implants, those who had the second implants had much better acceptance with less menstrual disturbances. International Journal of Gynaecology and Obstetrics 2000; 68: 249-56.

\section{Aminophylline in severe birth hypoxia}

Hypoxic ischaemic encephalopathy after severe birth hypoxia has been treated with drugs to reduce cerebral oedema (osmotic diuretics, dexamethasone and barbiturates). A recent study using Doppler ultrasound has shown that there is cerebral hyperfusion in the first 24 to 48 hours after birth in compromised newborns. In a small study of 9 severely asphyxiated newborns a bolus dose of aminophylline in a dose of $4 \mathrm{mg} / \mathrm{kg}$ produced sustained reductions in mean systolic and end-diastolic blood flow velocities and increases in mean pulsatility index. Presently it is not possible to decide whether the above documented changes are of any benefit to the infant. Further research is needed on this topic. Acta Paediatrica 2000; 89: 971-4.

R L Jayakody, Senior Lecturer, Department of Pharmacology, Faculty of Medicine, University of Colombo.

\section{Doctors are influenced by pharmaceutical representation (2)}

\begin{abstract}
Increasingly, undergraduate medical courses provide training including role-play to help future prescribers understand and perhaps profit more from seeing representatives. Given the significance of detailing to prescriber education, perhaps more attention needs to be paid to equipping current prescribers to deal more effectively with detailers.
\end{abstract}

Day R. How to make the most of a visit from a pharmaceutical company representative. Australian Prescriber 2000; 23: 97-9. 